\title{
One-dimensional Potts model, Lee-Yang edges, and chaos
}

\author{
Brian P. Dolan ${ }^{1,2}$ and D. A. Johnston ${ }^{3}$ \\ ${ }^{1}$ Department of Mathematical Physics, National University of Ireland, Maynooth, Ireland \\ ${ }^{2}$ School of Theoretical Physics, Dublin Institute for Advanced Studies, 10 Burlington Road, Dublin, Ireland \\ ${ }^{3}$ Department of Mathematics, Heriot-Watt University, Edinburgh EH14 4AS, Scotland
}

(Received 22 February 2002; published 14 May 2002)

\begin{abstract}
It is known that the exact renormalization transformations for the one-dimensional Ising model in a field can be cast in the form of the logistic map $f(x)=4 x(1-x)$ with $x$ a function of the Ising couplings $K$ and $h$. The locus of the Lee-Yang zeros for the one-dimensional Ising model in the $K, h$ plane is given by the Julia set of the logistic map. In this paper we show that the one-dimensional $q$-state Potts model for $q \geqslant 1$ also displays such behavior. A suitable combination of couplings, which reduces to the Ising case for $q=2$, can again be used to define an $x$ satisfying $f(x)=4 x(1-x)$. The Lee-Yang zeros no longer lie on the unit circle in the complex $z=e^{h}$ plane for $q \neq 2$, but their locus still maps onto the Julia set of the logistic map.
\end{abstract}

DOI: 10.1103/PhysRevE.65.057103

PACS number(s): 05.50. $+\mathrm{q}$

\section{INTRODUCTION: ISING MODEL}

Yang and Lee [1,2], later followed by various other authors [3], provided an important paradigm for understanding the nature of phase transitions by looking at the behavior of spin models in complex external fields. They observed that the partition function of a system above its critical temperature $T_{c}$ was nonzero throughout some neighborhood of the real axis in the complex external field plane. As $T \rightarrow T_{c}^{+}$the end points of loci of zeros moved in to pinch the real axis, signalling the transition. When such end points occur at nonphysical (i.e., complex) external field values they can be considered as ordinary critical points with an associated edge critical exponent. This appealing picture was later extended by Fisher to temperature driven transitions [4].

On any finite graph $G_{n}$ with $n$ vertices the free energy of an Ising-like spin model can be written as

$$
F\left(G_{n}, \beta, z\right)=-n h-\ln \prod_{k=1}^{n}\left[z-z_{k}(\beta)\right],
$$

where the fugacity $z=\exp (h)$, and $h$ is the (possibly complex) external field. The $z_{k}(\beta)$ are the Lee-Yang zeros, which in the thermodynamic limit often condense on curves in the complex $z$ plane. In the infinite volume limit $n \rightarrow \infty$ the free energy per spin is

$$
F\left(G_{\infty}, \beta, z\right)=-h-\int_{-\pi}^{\pi} d \theta \rho(\beta, \theta) \ln \left(z-e^{i \theta}\right),
$$

where $\rho(\beta, \theta)$ is the density of the zeros, which can be shown to appear on the unit circle in the complex $z$ plane in the Ising case (the Lee-Yang circle theorem). For $T>T_{c}$ or, if one prefers $\beta<\beta_{c}$, there is a gap with $\rho(\beta, \theta)=0$ for $|\theta|$ $<\theta_{0}$, and at these edge singularities we have

$$
\rho(\beta, \theta) \sim\left(\theta-\theta_{0}\right)^{\sigma}
$$

which defines the Lee-Yang edge singularity exponent $\sigma$. This also implies $M \sim\left(\theta-\theta_{0}\right)^{\sigma}$. Various finite size scaling relations relate the Lee-Yang exponent to the other critical exponents [5].

At first sight there is no apparent reason why the LeeYang edge singularity should bear any relation to the onset of chaotic behavior and Julia sets for nonlinear maps. However, the relation is well known in the case of spin models on hierarchical (fractal) lattices [6], where exact renormalization group ( $\mathrm{RG}$ ) transformations exist. It was realized that the Julia sets of the renormalization group transformations gave the boundaries of the basins of attractions (i.e., complex extended phases) of the high and low temperature attractors in the complex temperature plane and hence determined the loci of partition function zeros for such models. The idea has also been applied using an approximate renormalization group transformation to the two-dimensional (2D) Ising model on a square lattice and the two circles of Fisher zeros were recovered [7]. This picture of partition function zeros arising as complex temperature or field phase boundaries also ties in nicely with the recent work of Biskup et al. [8] who investigated Lee-Yang singularities in a general class of models with first-order transitions.

In this paper we look at another class of model with an exact renormalization group transformation, the 1D Potts model, and show that the Julia set of this transformation also gives the partition function zeros. The work reported in this paper extends the earlier observations of one of the authors on the 1D Ising model in Ref. [9], which we now briefly review for completeness. The partition function for the $1 \mathrm{D}$ Ising model is given by

$$
Z_{N}(K, h)=\sum_{\{\sigma\}} \exp \left[K \sum_{j=1}^{N} \sigma_{j} \sigma_{j+1}+h \sum_{j=1}^{N} \sigma_{j}\right],
$$

where $K=J / k T$ and $h=H / k T$, with $J$ the spin coupling and $H$ the external magnetic field, and periodic boundary conditions require $\sigma_{N+1} \equiv \sigma_{1}$. The well-known solution to the 1D Ising model proceeds by expressing $Z_{N}(K, h)$ in terms of the transfer matrix $V$ as $Z_{N}=\operatorname{Tr} V^{N}$, where 


$$
V(K, h)=\left(\begin{array}{cc}
V_{++} & V_{+-} \\
V_{-+} & V_{--}
\end{array}\right)=\left(\begin{array}{cc}
e^{K+h} & e^{-K} \\
e^{-K} & e^{K-h}
\end{array}\right) .
$$

Diagonalizing $V$ gives the eigenvalues $\lambda_{ \pm}=e^{K}\{\cosh h$ $\left.\pm \sqrt{\sinh ^{2} h+e^{-4 K}}\right\}$ and allows us to express the partition function as

$$
Z_{N}=\lambda_{+}^{N}+\lambda_{-}^{N} .
$$

The Lee-Yang zeros of this partition function in the complex $h$ plane are the $N$ roots of $Z_{N}(K, h)=0$, which are for real $K$ the solutions of

$$
Z_{N}=\left(\lambda_{+}\right)^{N}+\left(\lambda_{-}\right)^{N}=0 \Leftrightarrow \lambda_{+}=\exp \left(\frac{i n \pi}{N}\right) \lambda_{-},
$$

where $-N<n \leqslant N$ is odd. This gives the $N$ Lee-Yang zeros $h_{n}=i \theta_{n}$,

$$
\cos \left(\frac{n \pi}{2 N}\right) \sqrt{e^{-4 K}+\sinh ^{2}\left(h_{n}\right)}=i \sin \left(\frac{n \pi}{2 N}\right) \cosh \left(h_{n}\right) .
$$

We can see that when $K \rightarrow \infty$ (the zero temperature "transition point" for the 1D Ising model) the zeros are uniformly distributed on the unit circle in the complex $z=e^{h}$ plane, as demanded by the Lee-Yang theorem.

So far, so standard. Now note that the recursive renormalization group transformation for the 1D Ising model can be obtained by demanding that any renormalized couplings $K^{\prime}$ and $h^{\prime}$ satisfy

$$
Z_{N / 2}\left(K^{\prime}, h^{\prime}\right)=A^{N} Z_{N}(K, h),
$$

where $A$ is some renormalization factor. Thinking in terms of a decimation-type renormalization scheme it is clear that we can satisfy this by taking

$$
V\left(K^{\prime}, h^{\prime}\right)=A^{2} V(K, h)^{2},
$$

where $V$ is the transfer matrix given in Eq. (5). Viewed geometrically, we are welding two line segments together and demanding a suitable rescaling of the couplings, so one copy of the rescaled transfer matrix $V\left(K^{\prime}, h^{\prime}\right)$ must serve in place of two copies of the original $V(K, h)$. This leads to the recursion relations

$$
\begin{aligned}
e^{2 h^{\prime}} & =e^{2 h} \frac{\cosh (2 K+h)}{\cosh (2 K-h)}, \\
e^{4 K^{\prime}} & =\frac{\cosh (4 K)+\cosh (2 h)}{2 \cosh ^{2}(h)} .
\end{aligned}
$$

The crucial observation of Ref. [9] was that these recursion relations could be recast by making use of the renormalization invariant $m=1+e^{4 K} \sinh ^{2}(h)$ to eliminate $h$ and introducing the variable

$$
x=-\frac{m}{\left(e^{4 K}-1\right)}
$$

to transform Eq. (11) into the logistic map $x^{\prime}=4 x(1-x)$. This will exhibit chaotic behavior for $0<x<1$, i.e., if $m$ $=1+e^{4 K} \sinh ^{2}(h)<0$ which for imaginary external field, $h$ $=i \theta$, will occur if $\sin ^{2}(\theta)>e^{-4 K}$.

What has this got to do with Lee-Yang edge singularities? Looking back at Eq. (8) we can see that the lowest Lee-Yang zero will lie at $\sin ^{2}\left(\theta_{0}\right)=e^{-4 K}$, which is precisely the "boundary of chaos," $m=0$, in $x$ observed in the renormalization transformation above. One can also identify a gap exponent for the chaotic map that is identical to the Lee-Yang exponent $\sigma=-1 / 2$ for the 1D Ising model [9]. The example of hierarchical models where exact transformations also exist suggests that the identification of the Julia set of an RG transformation and the loci of partition function zeros, whether Lee-Yang edge singularities or Fisher zeros, is generic so it would be of interest to see other examples of the phenomenon. In the remainder of the paper we discuss just such an example, the 1D Potts model, where one can also construct an exact renormalization transformation along similar lines to the Ising model and obtain the Lee-Yang zeros explicitly.

\section{1D POTTS MODEL AND LEE-YANG ZEROS}

The partition function for the 1D Potts model is given by

$$
Z_{N}(y, z)=\sum_{\{\sigma\}} \exp \left[\widetilde{K} \sum_{j=1}^{N} \delta\left(\sigma_{j}, \sigma_{j+1}\right)+\widetilde{h} \sum_{j=1}^{N} \delta\left(\sigma_{j}, 1\right)\right],
$$

where the $\delta() \mathrm{s}$ are Kronecker deltas and there are now $q$ possible states for each spin $\sigma$. We have defined $y=e^{\widetilde{K}}$ and $z=e^{\tilde{h}}$ for later convenience. We can write down a transfer matrix for this as a $q \times q$ matrix $V(y, z)$ with $q-2$ diagonal elements $(y-1) /(y z)^{1 / q}$ and a $2 \times 2$ submatrix $T(y, z)[10]$

$$
T(y, z)=\frac{1}{(y z)^{1 / q}}\left(\begin{array}{cc}
y z & z^{1 / 2}(q-1) \\
z^{1 / 2} & y+q-2
\end{array}\right) .
$$

For $q=2$ we recover $V(K, h)$ from $T(y, z)$ providing we identify $\widetilde{K}=2 K, \widetilde{h}=2 h$.

The solution proceeds as in the Ising case by writing $Z_{N}(y, z)=\operatorname{tr} V(y, z)^{N}$ and diagonalizing $V$ [10]. The dominant eigenvalues $\lambda_{0,1}$ come from $T(y, z)$

$$
\begin{aligned}
\lambda_{0,1}= & \frac{1}{2}[(y(1+z)+q-2] \\
& \left. \pm \sqrt{[y(1-z)+q-2]^{2}+(q-1) 4 z}\right)(y z)^{-1 / q}
\end{aligned}
$$

which can be rewritten as

$$
\lambda_{0,1}=\frac{y}{2}\left(t_{+} t_{-}+z \pm \sqrt{\left(z-t_{+}^{2}\right)\left(z-t_{-}^{2}\right)}\right)(y z)^{-1 / q}
$$

with

$$
t_{ \pm}=\frac{1}{y}(\sqrt{(y-1)(y+q-1)} \pm \sqrt{1-q}) .
$$


The other $q-2$ eigenvalues given by $\lambda_{2}=\lambda_{3}=\ldots=(y$ $-1)(y z)^{-1 / q}$ play no role in the thermodynamic limit for $q$ $\geqslant 1$ as discussed in some detail in Ref. [10].

The Lee-Yang zeros $z_{n}=e^{h_{n}}$, as for the Ising model, appear as solutions of

$$
Z_{N}=\left(\lambda_{1}\right)^{N}+\left(\lambda_{0}\right)^{N}=0 \Leftrightarrow \lambda_{1}=\exp \left(\frac{\text { in } \pi}{N}\right) \lambda_{0},
$$

which, upon substituting in the values above for $\lambda_{0,1}$, gives

$$
\cos \left(\frac{n \pi}{2 N}\right) \sqrt{\left(z_{n}-t_{+}^{2}\right)\left(z_{n}-t_{-}^{2}\right)}=i \sin \left(\frac{n \pi}{2 N}\right)\left(t_{+} t_{-}+z_{n}\right),
$$

which is clearly of the same form as the Ising result in Eq. (8) for general $q$ and reproduces it exactly when $q=2$ (and we set $\widetilde{K}=2 K, \widetilde{h}=2 h$ ), as it should. The resemblance runs deeper even for general $q$, as noted in Ref. [10]. If we define $\tilde{z}=z /\left(t_{+} t_{-}\right)=y z /(y+q-2)$ the Lee-Yang zeros are again uniformly distributed round the unit circle in the complex $\tilde{z}$ plane as $\widetilde{K} \rightarrow \infty$ and $t_{+} \rightarrow 1, t_{-} \rightarrow 1$.

We now pursue the same path to construct the renormalization group transformation for the Potts model as we did for the Ising model. We demand that the renormalized couplings $y^{\prime}$ and $z^{\prime}$ satisfy

$$
Z_{N / 2}\left(y^{\prime}, z^{\prime}\right)=A^{N} Z_{N}(y, z),
$$

which can be solved by taking $V\left(y^{\prime}, z^{\prime}\right)=A^{2} V(y, z)^{2}$, where $V$ is now the Potts transfer matrix. Since only $\lambda_{0,1}$ are playing any role in the thermodynamic limit we discard the remaining $q-2$ eigenvalues and concentrate our attentions on the submatrix $T$, by demanding $T\left(y^{\prime}, z^{\prime}\right)=A^{2} T(y, z)^{2}$.

We find the following recursion relations:

$$
\begin{aligned}
\frac{1}{\left(y^{\prime} z^{\prime}\right)^{1 / q}}\left(y^{\prime} z^{\prime}\right) & =\frac{A^{2}}{(y z)^{2 / q}}\left[y^{2} z^{2}+z(q-1)\right], \\
\frac{1}{\left(y^{\prime} z^{\prime}\right)^{1 / q}}\left(y^{\prime}+q-2\right) & =\frac{A^{2}}{(y z)^{2 / q}}\left[z(q-1)+(y+q-2)^{2}\right], \\
\frac{1}{\left(y^{\prime} z^{\prime}\right)^{1 / q}}\left(z^{\prime}\right)^{1 / 2} & =\frac{A^{2} z^{1 / 2}}{(y z)^{2 / q}}(z y+y+q-2),
\end{aligned}
$$

which can be used to eliminate $A$ giving

$$
\begin{aligned}
& \frac{y^{\prime} z^{\prime}}{y^{\prime}+q-2}=\frac{y^{2} z^{2}+z(q-1)}{(y+q-2)^{2}+z(q-1)}, \\
& \frac{\left(z^{\prime}\right)^{1 / 2}}{y^{\prime}+q-2}=\frac{z^{1 / 2}(y z+y+q-2)}{(y+q-2)^{2}+z(q-1)} .
\end{aligned}
$$

It is then straightforward to show that, as for the Ising model, an invariant exists, in this case

$$
C=\frac{[y(1-z)+q-2]^{2}}{z},
$$

so we can use the expression for $C$ to eliminate $z$ and reduce our two recurrence relations to one for $y$ alone. Eliminating $z^{\prime}$ from Eq. (22) leads to a single recursion relation that can be written as

$$
y^{\prime}\left(y^{\prime}+q-2\right)-(q-1)=\frac{z[y(y+q-2)-(q-1)]^{2}}{[y(z+1)+q-2]^{2}} .
$$

Now we can use Eq. (23) to write

$$
C+4 y(y+q-2)=\frac{[y(z+1)+q-2]^{2}}{z} .
$$

So we define

$$
x=-\frac{[(C / 4)+q-1]}{[(y-1)(y+q-1)]}
$$

and the relation (24) is again reduced to the logistic map with the prefactor 4 ,

$$
x^{\prime}=4 x(1-x) .
$$

For $C$ real and positive $x$ is real and negative and so is outside the domain of chaos, but for $C<-4(q-1) x$ is positive we have chaos for $0<x<1$. On the critical line itself, $C$ $=-4(q-1)$, we allow ourselves the possibility of complex $z=|z| e^{i \theta}$ and find from Eq. (23) that

$$
z=\frac{(y+q-2)}{y} e^{i \theta}=\left(t_{+} t_{-}\right) e^{i \theta},
$$

where

$$
\cos (\theta)=1-2 \frac{(q-1)}{y(y+q-2)} .
$$

These are precisely the equations defining the Lee-Yang edge singularity in the 1D Potts model.

We have thus seen that defining a decimation-type renormalization transformation for the 1D Potts model gives rise to a set of recursion relations that may be reduced using the renormalization invariant of Eq. (23) to a single equation. This may in turn be mapped on to the logistic equation. The boundary of the chaotic region for this logistic map is identical to the critical line of the Lee-Yang edge singularity. This behavior is entirely analogous to that seen in the 1D Ising model in Ref. [9] and in the hierarchical models that also possess an exact renormalization group transformation.

\section{DISCUSSION}

The similarity of the Lee-Yang edge singularity for the general $q$ state Potts models in 1D and for the Ising model was already remarked in Ref. [10]. The exponent $\sigma=-1 / 2$ is identical for all $q>1$, and in suitably rescaled variables the 
Lee-Yang zeros lie on the unit circle at $T=0$ for all $q>1$. In this paper we have seen that a similar construction of an exact renormalization transformation may be employed in both the 1D Potts and 1D Ising models and that in both cases the Julia set of the mapping gives the Lee-Yang singularity.

That this should be so is in accordance with both earlier observations of spin models on hierarchical lattices [6] and the idea in Ref. [8] that loci of partition function zeros can profitably be thought of as phase boundaries in the complex temperature or field planes. Just as in the hierarchical lattice models, the Julia set of the renormalization map forms the boundary between the different basins of attraction, or phases, and hence coincides with the locus of zeros of the partition function.

An interesting counterpoint to these results is provided by the very detailed analysis of real-space renormalization group flow in $q$-state clock models with an additional imaginary interaction that was carried out in Ref. [11]. Without the imaginary term the three-state clock model is equivalent to the three-state Potts model and displays only the zero temperature continuous transition, but the addition of the imaginary term allows first order transitions with nongeneric features [12]. The Lee-Yang zeros we discuss here arise essentially by looking for the equality (in modulus) of two of the transfer matrix eigenvalues, whilst extending the zero temperature transition point to the Lee-Yang line by complexifying the external field.

It was noted in Ref. [11] that "Lee-Yang-Type" singularities in the clock models could also arise within transition surfaces (where two of the eigenvalues were already equal) when elements of powers of the transfer matrix were zero or, equivalently, the eigenvalues degenerated in pairs. Although the net result is the same in both cases-a zero partition function - the mechanism by which this arises and the implications are rather different. In the clock models the LeeYang-type singularities are interpreted as indications of nonuniversal pathologies for certain real-space renormalization transformations for particular regions in parameter space, whereas the standard Lee-Yang singularity is viewed more as standard critical behavior, albeit at a complex parameter value. The general possibilities for pathologies of approximate real space renormalization transformations within phases in higher-dimensional models were also discussed in a rigorous manner in Ref. [13].

Other cases exist where recursive nonlinear maps are used in the definition of exact partition functions, notably for spin models on Bethe lattices (trees). There has been discussion of chaotic effects in such models when $q<1$ [14] and the logistic equation has even been observed for a $q=1$ state Potts model related to percolation on a Bethe lattice with coordination number 3 [15].

\section{ACKNOWLEDGMENTS}

B.D. was partially supported by Enterprise Ireland Basic Research Grant No. SC/1998/739 and B.D. and D.J. by an Enterprise Ireland/British Council Research Visits Scheme Grant No. BC/2000/004. B.D. would like to thank HeriotWatt Mathematics Department for its hospitality and D.J. would like to thank the Department of Mathematical Physics, National University of Ireland, Maynooth for the same.
[1] T.D. Lee and C.N. Yang, Phys. Rev. 87, 410 (1952).

[2] C.N. Yang and T.D. Lee, Phys. Rev. 87, 404 (1952).

[3] J. Lebowitz and O. Penrose, Commun. Math. Phys. 11, 99 (1968); G. Baker, Phys. Rev. Lett. 20, 990 (1968); R. Abe, Prog. Theor. Phys. 37, 1070 (1967); 38, 72 (1967); 38, 568 (1967); S. Ono, Y. Karaki, M. Suzuki and C. Kawabata, J. Phys. Soc. Jpn. 25, 54 (1968); D. Gaunt and G. Baker, Phys. Rev. B 1, 1184 (1970); P. Kortman and R. Griffiths, Phys. Rev. Lett. 27, 1439 (1971); M. Fisher, ibid. 40, 1610 (1978).

[4] M. Fisher, in Lectures in Theoretical Physics (University of Colorado Press, Boulder, 1965), Vol. VII.

[5] C. Itzykson, R. Pearson, and J. Zuber, Nucl. Phys. B 220, 415 (1983).

[6] B. Derrida, L. De Seze, and C. Itzykson, J. Stat. Phys. 33, 559 (1983); B. Derrida, C. Itzykson and J. Luck, Commun. Math. Phys. 94, 115 (1984); B. Derrida, J. Eckmann, and A. Erzan, J.
Phys. A 16, 893 (1983).

[7] B. Derrida and H. Flyvbjerg, J. Phys. A 18, L313 (1985).

[8] M. Biskup, C. Borgs, J.T. Chayes, L.J. Kleinwaks, and R. Kotecky, Phys. Rev. Lett. 84, 4794 (2000).

[9] B. Dolan, Phys. Rev. E 52, 4512 (1995); 53, 6590 (1996).

[10] Z. Glumac and K. Uzelac, J. Phys. A 27, 7709 (1994).

[11] M. Asorey, J.G. Esteve, and J. Salas, Phys. Rev. B 48, 3626 (1993); M. Asorey, J.G. Esteve, J. Fernandez, and J. Salas, Nucl. Phys. B 392, 593 (1993).

[12] M. Asorey and J.G. Esteve, J. Stat. Phys. 65, 483 (1991).

[13] A.C.D. van Enter, R. Fernandez, and A.D. Sokal, J. Stat. Phys. 72, 879 (1994).

[14] J.L. Monroe, J. Phys. A 29, 5421 (1996).

[15] F. Wagner, D. Grensing, and J. Heide, J. Phys. A 33, 929 (2000). 